\title{
Possible Explanation of the Dynamics of the Universe Expansion without Dark Energy and without New Gravitational Degrees of Freedom
}

\author{
Eugene 0ks \\ Physics Department, Auburn University, Auburn, USA \\ Email: goks@physics.auburn.edu
}

How to cite this paper: Oks, E. (2021) Possible Explanation of the Dynamics of the Universe Expansion without Dark Energy and without New Gravitational Degrees of Freedom. Open Journal of Microphysics, 11, 1-7.

https://doi.org/10.4236/ojm.2021.111001

Received: February 6, 2021

Accepted: February 25, 2021

Published: February 28, 2021

Copyright ( 2021 by author(s) and Scientific Research Publishing Inc. This work is licensed under the Creative Commons Attribution International License (CC BY 4.0).

http://creativecommons.org/licenses/by/4.0/

\begin{abstract}
The commonly accepted view is that the Universe is currently in the dark energy dominance era (estimated to start about 5 billion years ago)-the era where yet unknown dark energy dominates over the gravitation and is responsible for the observed acceleration of the Universe expansion. In the present paper, we consider a "gas" of a large number of gravitating neutral nonrelativistic particles having a practically infinite lifetime and zero or very little interaction with the rest of the matter (neutrinos could be an example). One of the central points is the application of Dirac's Generalized Hamiltonian Dynamics to pairs of these particles. Another central point is the application of the virial theorem to pairs of zero total energy. We demonstrate that as a result, the gravitational interaction within the entire system effectively decreases. Together with the observational fact of the Universe rotation (according to Shamir's study of 2020), this model provides a possible explanation of the entire history of the Universe expansion: both the era of the decelerating expansion and the current era of the accelerated expansion.
\end{abstract}

\section{Keywords}

Dynamics of the Universe Expansion, No Dark Energy Required, No Massive Gravity Required

\section{Introduction}

Currently, the two greatest mysteries in physics seem to be dark matter and dark energy. Almost all models of dark matter resort to largely-unspecified never-discovered subatomic particles or introduce new physical laws [1]-[8], and references therein. One exception is papers showing the possibility that dark 
matter (or at least a part of it) can consist of hydrogen atoms of the 2nd flavor, whose existence is proven by the analysis of atomic experiments and can also explain the latest observations of $21 \mathrm{~cm}$ radio line from the early Universe [9] [10].

As for dark energy, there are lots of field and particle candidates for it (in addition again to suggestions to introduce new gravitational degrees of freedom, of which massive gravity is one of the examples), but these candidates have never been discovered so far [11]-[16], and references therein. This is even more intriguing because the commonly accepted view is that the Universe is currently in the dark energy dominance era (estimated to start about 5 billion years ago)-the era where yet unknown dark energy dominates over the gravitation and is responsible for the observed acceleration of the Universe expansion.

In the present paper, we introduce a scenario providing a possible explanation of the entire history of the Universe expansion: both the era of the decelerating expansion and the current era of the accelerated expansion. In this model, there is no need to resort to dark energy or to new gravitational degrees of freedom (mass gravity etc.).

\section{Results}

We present this scenario as the following sequence of logical steps.

1) We consider a "gas" of a large number of gravitating neutral nonrelativistic particles of mass $\mathrm{m}$. The corresponding Hamiltonian is:

$$
H=\sum_{i} p_{i}^{2} /(2 m)-\sum_{i \neq j} G m^{2} /\left|\boldsymbol{r}_{i}-\boldsymbol{r}_{j}\right|,
$$

where $\boldsymbol{r}_{i}$ and $\boldsymbol{p}_{i}$ are the radius-vector and the momentum of particle number $i$, respectively; $G$ is the gravitational constant.

At any instant of time, the gas has a subsystem of relatively isolated pairs of particles: a pair of particles is considered relatively isolated if their separation is much smaller than their distance to other particles. The subsystem is open: in the course of time, some pairs could stop qualifying as the subsystem members (because they can no more be considered as relatively isolated), while some other pairs could become relatively isolated and qualify as new members of the subsystem. Here the word "subsystem" does not mean a localized part of the entire system: rather it means a subset of the particle within the ensemble-the subset of pairs (not located in one particular volume) that are relatively isolated.

2) Pairs of the positive total energy $(E>0)$ lose the energy by the gravitational radiation and reach the state of $E=0$. For clarity: we consider non-relativistic particles and by their total energy we mean the difference between the relativistic total energy and the rest mass multiplied by the square of the speed of light.

3) To the motion of pairs of $E=0$ applies the virial theorem-contrary to many textbooks stating that the virial theorem applies only to bound states. As shown by Pollard [17], the boundness of a state is not the necessary condition for the applicability of the virial theorem. He derived a more general condition 
that is both necessary and sufficient. He specifically showed that the virial theorem applies to pairs of $E=0$ interacting by Newton's gravity.

4) Therefore, the subsystem of pairs of $E=0$ is characterized by the following values of the average potential $(\langle U\rangle)$ and kinetic $(\langle K\rangle)$ energies: $\langle U\rangle=\langle K\rangle=0$.

5) Now we apply Dirac's Generalized Hamiltonian Dynamics (GHD) to the above subsystem of pairs. Dirac designed the GHD for applications to quantum field theory [18] [19] [20]. The central point of Dirac's GHD is the addition of a linear combination of constraints to the classical Hamilton function. A brief description of Dirac's GHD and of its further development is presented in Appendix A.

Oks and Uzer [21] demonstrated how to apply Dirac's GHD to microscopic systems of particles, such as e.g., atomic and molecular systems. Specifically, as constraints they used classical integrals of the motion. By doing so, they showed, e.g., that hydrogenic atoms/ions, being described by Dirac's GHD, exhibit a sequence of classical non-radiating states, i.e., states where the system does not emit the electromagnetic radiation-it is radiationally-stable. The set of energies of these classical non-radiating states turned out to exactly coincide with the corresponding quantum stationary states.

The most important feature of classical non-radiating stable states is the following. In such a state, the radius-vector of the atomic electron $\boldsymbol{r}(t)=\boldsymbol{r}_{0}$ and the generalized momentum $\boldsymbol{P}(t)=\boldsymbol{P}_{0}$, where $\boldsymbol{r}_{0}$ and $\boldsymbol{P}_{0}$ are some vector constants. Thus, the electron velocity $\boldsymbol{V}=0$, but its generalized momentum $\boldsymbol{P}_{0}$ generally differs from zero. This should not be shocking: the generalized momentum is a more complex physical quantity than the velocity. For instance, it is well-known that for a charge e of mass $\mathrm{m}$ in an electromagnetic field, characterized by a vector-potential $\boldsymbol{A}$, it is also possible to have $\boldsymbol{V}=\boldsymbol{P} / m-e \boldsymbol{A} /(m c)=0$ while $\boldsymbol{P}=e \boldsymbol{A} / \mathrm{c}$ $\neq 0$ [22], the formula after Equation (16.9).

6) The way Oks \& Uzer [21] applied Dirac's GHD to pairs of charges interacting by the Coulomb law can be similarly applied to pairs of neutral particles interacting by Newton's gravity. The result is the infinite number of classical non-radiating states of the energies

$$
E_{k}=-B / k^{2}, \quad B=\text { const }>0, \quad k=1,2,3, \cdots, \infty .
$$

Here $k$ is the harmonic number of the primary frequency of the particle moving along an elliptical trajectory. It is convenient to denote $q=1 / k$, so that

$$
E_{q}=-B q^{2}, q=0, \cdots, 1 .
$$

It is obvious that $q$ takes the infinite amount of rational numbers in the segment $[0,1]$ and that $E_{q=0}=0$.

7) Thus, within Dirac's GHD, the pairs of gravitating neutral particles of energies $E>0$, lose the energy by the gravitational radiation, reach the state of $E=0$, and in this state the gravitational radiation vanishes. The subsystem of pairs of $E$ $=0$ is radiationally stable. 
8) While the subsystem of pairs of $E=0$ has $\langle K\rangle=0$, the rest of the gas has $\langle K\rangle_{\text {rest }}>0$ (the subscript "rest" stands for the "rest of gas"). The two subsystems interact and can exchange members. Therefore, the entire system tends to thermalize (due to the exchange of members of the two subsystems), so that for the entire system we have the average kinetic energy $\langle K\rangle\left\langle\langle K\rangle_{\text {rest }}\right.$.

9) The average kinetic energy of the rest of the gas $\langle K\rangle_{\text {rest }}$ will continue to decrease in the course of time so that the entire gas will have the average kinetic energy $\langle K\rangle \rightarrow 0$. This means that in the course of time, practically the entire gas would become thermodynamically equivalent to a set of non-radiating pairs of $E=0$.

10) Here we come to the following central point. The "freezing" of the pairs into classical states of $\boldsymbol{r}=$ const is equivalent to a partial inhibition of the gravitation. Indeed, without it, the gravitational radiation and the loss of the total energy would continue with $100 \%$ effectiveness and the pairs would relatively rapidly collapse (being considered classically). However, the partial inhibition of the gravitation slows down this process.

11) Thus, as the share of the subsystem of pairs (in the entire system) increases in the course of time, the gravitational interaction within the entire system effectively decreases and tends to zero.

12) For this scenario, the particles of the gas should have a practically infinite lifetime and should have zero or very little interaction with the rest of the matter. Therefore, the most probable candidate for these particles is non-relativistic neutrinos. The current technology allows detecting only relativistic neutrinos-because slow-moving neutrinos have very low probabilities of interactions with the rest of the matter. However, nothing prohibits neutrinos from moving at non-relativistic velocities.

13) According to the recent study by Shamir [23], there is a disparity between galaxies spinning clockwise and counterclockwise so that the Universe as the whole rotates. Thus, there is a centrifugal force. After the gravitation would get partially inhibited at the above stage 10, the centrifugal force would start dominating over the gravitation and the particles of the rotating gas would expand at an increasing speed.

\section{Conclusions}

We considered a "gas" of a large number of gravitating neutral particles. We presented a step-by-step scenario, one of the central points of which was the application of Dirac's GHD to pairs of these particles. Another central point was that the virial theorem is valid for applies to pairs of zero total energy interacting by Newton's gravity-contrary to many textbooks stating that the virial theorem applies only to bound states. We showed that as a result, the gravitational interaction within the entire system effectively decreases.

The following point should be emphasized. Just the Universe rotation alone cannot explain why in the first 9 billion years or so, the expansion of the Un- 
iverse was decelerating. The entire situation can be explained by the combination of the inhibition of the gravitational interaction (described above) complemented by the rotation of the Universe. In other words, it took time for the gas to reach the above stage 10 (the "freezing" of the gravitation). During this time, the gravitation was still dominating the opposing centrifugal force caused by the rotation of the Universe, so that the Universe expansion was slowing down. Then, after the gas reached the above stage 10, the centrifugal force, caused by the Universe rotation, started dominating the gravitation, so that the Universe expansion started to accelerate.

Thus, in this model, there is no need to resort to dark energy or to new gravitational degrees of freedom (mass gravity, etc.) for explaining the entire history of the Universe expansion, including the observed acceleration of the expansion in the last 5 billion years or so.

\section{Conflicts of Interest}

The author declares no conflicts of interest regarding the publication of this paper.

\section{References}

[1] de Martino, I., Chakrabarty, S.S., Cesare, V., Gallo, A., Ostorero, L. and Diaferio, A. (2020) Dark Matters on the Scale of Galaxies. Universe, 6, Article No. 107. https://doi.org/10.3390/universe6080107

[2] Salucci, P., Turini, N. and di Paolo, C. (2020) Paradigms and Scenarios for the Dark Matter Phenomenon. Universe, 6, Article No. 118.

https://doi.org/10.3390/universe6080118

[3] Bertone, G. and Hooper, D. (2018) History of Dark Matter. Reviews of Modern Physics, 90, Article No. 045002. https://doi.org/10.1103/RevModPhys.90.045002

[4] Bertone, G. and Tait, T.M.P. (2018) A New Era in the Search for Dark Matter. Nature, 562, 51-56. https://doi.org/10.1038/s41586-018-0542-z

[5] Einasto, J. (2013) Dark Matter and Cosmic Web Story. World Scientific, Singapore. https://doi.org/10.1142/8928

[6] Majumdar, D. (2014) Dark Matter: An Introduction. CRC Press, Boca Raton, USA. https://doi.org/10.1201/b17323

[7] Garrett, K. and Düda, G. (2011) Dark Matter: A Primer. Advances in Astronomy, 2011, Article ID: 968283. https://doi.org/10.1155/2011/968283

[8] Sanders, R.H. (2010) The Dark Matter Problem: A Historical Perspective. Cambridge University Press, Cambridge, England. https://doi.org/10.1017/CBO9781139192309

[9] Oks, E. (2020) Alternative Kind of Hydrogen Atoms as a Possible Explanation of the Latest Puzzling Observation of the $21 \mathrm{~cm}$ Radio Line from the Early Universe. $R e$ search in Astronomy and Astrophysics, 20, Article No. 109. https://doi.org/10.1088/1674-4527/20/7/109

[10] Oks, E. (2020) Two Flavors of Hydrogen Atoms: a Possible Explanation of Dark Matter. Atoms, 8, Article No. 33. https://doi.org/10.3390/atoms8030033

[11] Frusciante, N. and Perenon, L. (2020) Effective Field Theory of Dark Energy: A Review. Physics Reports, 857, 1-63. https://doi.org/10.1016/j.physrep.2020.02.004 
[12] Tawfik, A.N. and Dahab, E.A.E. (2019) Review on Dark Energy Models. Gravitation and Cosmology, 25, 103-115. https://doi.org/10.1134/S0202289319020154

[13] Brax, P. (2017) What Makes the Universe Accelerate? A Review on What Dark Energy Could Be and How to Test It. Reports on Progress in Physics, 81, Article No. 016902. https://doi.org/10.1088/1361-6633/aa8e64

[14] Joyce, A., Lombriser, L. and Schmidt, F. (2016) Dark Energy versus Modified Gravity. Annual Review of Nuclear and Particle Science, 66, 95-122. https://doi.org/10.1146/annurev-nucl-102115-044553

[15] Kenath, A., Gudennavar, S.B. and Sivaram, C. (2017) Dark Matter, Dark Energy, and Alternate Models: A Review. Advances in Space Research, 60, 166-186. https://doi.org/10.1016/j.asr.2017.03.043

[16] Starkman, G.D. (2011) Modifying Gravity: You Cannot Always Get What You Want. Philosophical Transactions of the Royal Society A, 369, 5018-5041. https://doi.org/10.1098/rsta.2011.0292

[17] Pollard, H. (1964) A Sharp Form of the Virial Theorem. Bulletin of the American Mathematical Society, 70, 703-705. https://doi.org/10.1090/S0002-9904-1964-11175-7

[18] Dirac, P.A.M. (1950) Generalized Hamiltonian Dynamics. Canadian Journal of Mathematics, 2, 129-148. https://doi.org/10.4153/CJM-1950-012-1

[19] Dirac, P.A.M. (1958) Generalized Hamiltonian Dynamics. Proceedings of the Royal Society A, 246, 326-332. https://doi.org/10.1098/rspa.1958.0141

[20] Dirac, P.A.M. (1964) Lectures on Quantum Mechanics. Academic Press, New York.

[21] Oks, E. and Uzer, T. (2002) Application of Dirac's Generalized Hamiltonian Dynamics to Atomic and Molecular Systems. Journal of Physics B: Atomic, Molecular and Optical Physics, 35, 165-173. https://doi.org/10.1088/0953-4075/35/1/312

[22] Landau, L.D. and Lifshitz, E.M. (1975) Classical Theory of Fields. Pergamon Press, Oxford.

[23] Shamir, L. (2020) Patterns of Galaxy Spin Directions in SDSS and Pan-STARRS Show Parity Violation and Multipoles. Astrophysics and Space Science, 365, Article No. 136. https://doi.org/10.1007/s10509-020-03850-1

[24] Oks, E. (2015) Breaking Paradigms in Atomic and Molecular Physics. World Scientific, Singapore. https://doi.org/10.1142/9301

[25] Oks, E. (2019) Unexpected Similarities of the Universe with Atomic and Molecular Systems: What a Beautiful World. IOP Publishing, Bristol, UK, Appendix A. https://doi.org/10.1088/2053-2563/aafed9

[26] Loeb, L.L. (1938) Atomic Structure. Wiley \& Sons, London.

[27] Camarena, A. and Oks, E. (2010) Application of the Generalized Hamiltonian Dynamics to a Modified Coulomb Potential. Intern. International Review of Atomic and Molecular Physics, 1, 143-160.

[28] Oks, E. (2020) Application of the Generalized Hamiltonian Dynamics to Spherical Harmonic Oscillators. Symmetry, 12, Article No. 1130.

https://doi.org/10.3390/sym12071130 


\section{Appendix A. Brief Description of the Application of Dirac's Generalized Hamiltonian Dynamics (GHD) to Microscopic Systems of Particles}

The central point of Dirac's GHD is the introduction of a classical generalized Hamilton function

$$
H_{g}(q, p)=H(q, p)+u_{m} \phi_{m}(q, p), \quad m=1,2, \cdots, M,
$$

where $H(q, p)$ is the conventional classical Hamilton function and the $2^{\text {nd }}$ term is a linear combination of constraints (Dirac [18] [19] [20]). Here and below, the summation over twice repeated suffix is understood. The coefficients $u_{m}$ can be determined from so-called consistency conditions

$$
\left[\phi_{m^{\prime}}, H\right]+u_{m}\left[\phi_{m^{\prime}}, \phi_{m}\right] \approx 0 . \quad m^{\prime}=1,2, \cdots, M,
$$

where $[f, g]$ is the usual Poisson bracket. (To distinguish equations that hold after the variation of dynamical variables from equations that do not hold, Dirac used the sign "=" for the former and the sign " $\approx$ " for the latter.)

[21] further developed Dirac's GHD by using classical integrals of the motion as constraints [24] [25]. Specifically, for hydrogenic atoms/ions they used components of the angular momentum and of the Runge-Lenz vector as the constraints. This turned out to be sufficient for proving the existence of classical non-radiating states.

For obtaining further results, Oks \& Uzer [21] employed Plank's hypothesis (later incorporated by Einstein into his photoelectric law [26]), which can be formulated as follows. In the process of the radiation, the change of the energy between the initial and final states $\Delta \mathrm{E}$ should be proportional to the average frequency of the radiation $\langle\omega\rangle$ in this process. As a result, Oks \& Uzer obtained the expression for the infinite set of classical non-radiating states $E_{k}$ (where $k=1,2,3, \cdots, \infty)$ coinciding with the corresponding set of quantum stationary states.

Later on, Dirac's GHD was successfully applied to other microscopic systems and to finding classical non-radiating states in these systems. Example is pairs of particles interacting by a modified Coulomb potential [27] and a spherical harmonic oscillator [28]. 